\title{
Discours
}

Revue de linguistique, psycholinguistique et

informatique. A journal of linguistics, psycholinguistics and computational linguistics

$25 \mid 2019$

Varia

\section{La référence pronominale en français et en mandarin : une étude contrastive basée sur un corpus de traductions bidirectionnelles}

Juan Sun et Qiang Zhang

\section{OpenEdition}

Journals

Édition électronique

URL : http://journals.openedition.org/discours/10579

DOI : 10.4000/discours. 10579

ISSN : 1963-1723

Éditeur :

Laboratoire LATTICE, Presses universitaires de Caen

\section{Référence électronique}

Juan Sun et Qiang Zhang, «La référence pronominale en français et en mandarin : une étude contrastive basée sur un corpus de traductions bidirectionnelles », Discours [En ligne], 25 | 2019, mis en ligne le 30 décembre 2019, consulté le 04 avril 2020. URL : http://journals.openedition.org/ discours/10579; DOI : https://doi.org/10.4000/discours.10579 

Revue de linguistique, psycholinguistique et informatique

\title{
La référence pronominale en français et en mandarin : une étude contrastive basée sur un corpus de traductions bidirectionnelles
}

\author{
Juan Sun \\ Université Sun Yat-sen \\ Qiang Zhang \\ Université du Shandong
}





\title{
La référence pronominale en français et en mandarin : une étude contrastive basée sur un corpus de traductions bidirectionnelles
}

\author{
Juan Sun \\ Université Sun Yat-sen \\ Qiang Zhang \\ Université du Shandong
}

Cette étude empirique basée sur corpus vise à examiner de manière quantitative la question de la référence pronominale dans deux langues typologiquement différentes et géoculturellement éloignées: le français et le chinois mandarin. En effectuant des analyses monolingues et interlinguistiques sur un corpus parallèle composé de textes authentiques traduits du français vers le mandarin ou dans la direction inverse, nous mettons en évidence une disparité importante entre ces deux langues dans le choix des moyens référentiels. Conformément à nos attentes, le français s'avère avoir une prédilection pour la pronominalisation, alors que le mandarin est caractérisé par une fréquence importante des reprises nominales et notamment des ellipses. Cette différence de choix référentiel est liée en général aux traits spécifiques du système pronominal de chaque langue et explique pour une grande part le contraste saisissant entre les tendances de traduction du français vers le mandarin et du mandarin vers le français.

Mots clés: référence pronominale, traductions, français, mandarin, corpus parallèle

This paper aims at a quantitative investigation on pronominal references in two typologically and geo-culturally distinct languages: French and Mandarin Chinese. By conducting monolingual and inter-linguistic analyses based on a parallel corpus consisting of authentic texts translated from French into Mandarin or in the opposite direction, we demonstrated that apropos of the choice of referential expressions there is a wide divergence between these two languages. In accordance with our expectations, it seems that French has a predilection for the use of pronominal references, whereas Mandarin is characterized by a high frequency of nominal repetitions and in particular of ellipses. This difference is in general linked to the specific features of the pronominal system in each of the two languages and explains to a large extent the striking contrast between the translation tendencies from French to Mandarin and from Mandarin to French.

Keywords: pronominal reference, translations, French, Mandarin, parallel corpus

Cette recherche a été menée dans le cadre du «Projet du $13^{e}$ plan quinquennal pour la philosophie et les sciences sociales du Guangdong» ( $n^{\circ}$ GD17XWW20) et du «Projet national de la Fondation en sciences sociales de la Chine» (n 17ZDA167). 


\section{Introduction}

L'abondante littérature consacrée à la référence pronominale dans le domaine de la linguistique témoigne de l'intérêt croissant qu'elle suscite. Les études portant sur ce phénomène linguistique sont multiples et hétérogènes dans la mesure où elles ont été menées selon différentes approches: l'approche syntactico-formelle de la grammaire générative (Zribi-Hertz, 1996), l'approche textuelle (Halliday et Hasan, 1976; Charolles, 1978 et 1995; Adam, 1999 et 2005), l'approche pragmatique (Reboul, 1989 et 1994; Reboul et Moeschler, 1998), l'approche (pragma-)sémantique (Kleiber, 1994; De Mulder et al., 2001), et l'approche cognitive (Ariel, 1988; Cornish, 2000), selon la distinction de Demol (2008; cité d'après Jonasson, 2009).

Dans le cadre d'une perspective contrastive, nombre d'études comme celles de Clancy (1980), de Lundquist (2005 et 2007), de Cornish et al. (2005), de Jonasson (2006 et 2009) et de Vanderbauwhede (2014) ont mis en évidence la variation interlinguistique due aux propriétés spécifiques de chaque langue. Par exemple, Clancy (1980) a souligné les fonctions différentes des formes référentielles semblables en anglais et en japonais. Les recherches de Lundquist (2005 et 2007) ont montré que la fréquence des références démonstratives est plus importante en français qu'en danois. Jonasson (2009) a observé que les anaphores pronominales sont plus présentes dans les textes en français qu'en suédois. Vanderbauwhede (2014) a signalé la divergence entre le français et le néerlandais à l'égard de l'anaphore démonstrative. Parmi les études contrastives, celles qui opposent deux ou plusieurs langues typologiquement différentes et à la fois fort éloignées, tant du point de vue géographique que culturel, sont relativement peu nombreuses. En outre, du point de vue méthodologique, malgré le nombre croissant des études empiriques, celles qui utilisent un corpus de traductions bidirectionnelles restent encore limitées. Ainsi, nous proposons cette étude empirique sur la référence pronominale dans les traductions bidirectionnelles entre le français et le chinois mandarin (ci-après dénommé mandarin). L'objectif est de fournir un aperçu quantitatif de la référence pronominale en français et en mandarin, de mesurer le degré de leur divergence dans le choix des moyens référentiels, et d'examiner l'influence de la langue source sur le choix référentiel dans les textes traduits.

Le corpus utilisé pour cette étude est composé de 45 textes bilingues de longueur différente, dont 30 sont traduits du mandarin vers le français et 15 sont traduits du français vers le mandarin. Au total, nous avons identifié 317 paires à étudier pour la direction du mandarin vers le français et 291 pour celle du français vers le mandarin. Les résultats obtenus sont présentés et discutés dans la section 3. Mais pour commencer, dans la section 2, nous soulignons quelques particularités du système pronominal chinois comparées à celles du français, permettant d'expliquer dans une certaine mesure les tendances différentes de choix référentiel dans les deux langues, avant de montrer les divers types de variations de traduction observés dans le corpus. 


\section{Référence pronominale dans les traductions entre le français et le mandarin}

Dans cette section, avec une attention particulière portée aux pronoms personnels, nous présentons d'abord les particularités du système pronominal chinois comparées à celles du français, et ensuite les divers types de variations observés dans le corpus.

\subsection{Systèmes pronominaux français et chinois}

5 Le système pronominal chinois est beaucoup moins riche et complexe comparé à celui du français, en dépit de quelques ressemblances apparentes. D'une part, le mandarin ne possède pas autant de types pronominaux que l'on peut en trouver en français (par exemple, le mandarin est dépourvu de pronoms relatifs), et d'autre part, étant une langue à flexion pauvre, le mandarin n'a qu'une liste de pronoms morphologiquement restreinte par rapport au français. Par exemple, à la différence du français dans lequel la forme des pronoms personnels varie selon la fonction qu'ils occupent dans la proposition, le mandarin utilise une même forme pour remplir différentes fonctions syntaxiques. Comme l'illustre le tableau 1 (inspiré de Grevisse et Goosse, 2008: 659), les diverses formes du pronom de la première personne du singulier en français $(j e, m e, m o i)$ peuvent toutes être traduites par 我 wó en mandarin.

\begin{tabular}{|c|c|c|c|c|c|c|}
\hline & & \multicolumn{4}{|c|}{ Français } & \multirow{3}{*}{ Mandarin } \\
\hline & & \multicolumn{3}{|c|}{ Formes conjointes } & \multirow{2}{*}{$\begin{array}{l}\text { Formes } \\
\text { disjointes }\end{array}$} & \\
\hline & & Sujet & Objet direct & Objet indirect & & \\
\hline \multicolumn{2}{|c|}{$1 \mathrm{SG}$} & je & \multicolumn{2}{|c|}{ me } & moi & 我 wǒ \\
\hline \multicolumn{2}{|c|}{$2 S G$} & tu & \multicolumn{2}{|c|}{ te } & toi & 你 $n \check{\imath}$ \\
\hline \multirow{2}{*}{$3 S G$} & Masc. & il & le & \multirow{2}{*}{ lui } & lui & 他/它 $t \bar{a}$ \\
\hline & Fém. & elle & la & & elle & 她/它 $t \bar{a}$ \\
\hline \multicolumn{2}{|c|}{ 1PL } & \multicolumn{4}{|c|}{ nous } & 我们 wǒmen \\
\hline \multicolumn{2}{|c|}{$2 \mathrm{PL}$} & \multicolumn{4}{|c|}{ vous } & 你们 nimmen \\
\hline \multirow{2}{*}{ 3PL } & Masc. & ils & \multirow{2}{*}{ les } & \multirow{2}{*}{ leur } & eux & 他/它们 tāmen \\
\hline & Fém. & elles & & & elles & 她/它们 tāmen \\
\hline
\end{tabular}

Tableau 1 - Formes du pronom personnel en français et leur équivalent en mandarin ${ }^{1}$

1. Il est à noter que ce tableau n'illustre que les pronoms personnels non réfléchis existant dans les deux langues et que les équivalents chinois proposés sont les plus conventionnellement utilisés (il y en a encore quelques autres comme le «nous» inclusif 咱们 zánmen et le «vous» de politesse 您 nín). 
En outre, le français et le mandarin diffèrent en ce qui concerne l'emploi des pronoms de la troisième personne. Comme nous pouvons le constater dans le tableau 1, le français fait la distinction entre masculin et féminin, alors que le mandarin possède trois formes (partageant la même prononciation) pour indiquer respectivement le masculin (他 $t \bar{a}$ ), le féminin (她 $t \bar{a}$ ) et le neutre (它 $t \bar{a}$ ). Les deux premières ne servent généralement qu'à désigner des personnes, tandis que la dernière est employée uniquement pour référer à des entités non humaines (animaux ou objets inanimés). En résumé, le mandarin fait deux distinctions par rapport au caractère humain ou non humain du référent: humain et non humain et, pour désigner un être humain, masculin et féminin. Strictement parlant, ces distinctions n'étaient pas inhérentes au système pronominal chinois, car elles ont été introduites dans la langue chinoise dans les années 1910, par le biais d'un «emprunt conceptuel» à l'anglais (voir Wang, 1954), représentant ainsi une des tentatives importantes au cours de la première moitié du XX siècle d'européaniser la grammaire chinoise. Pourtant, malgré cela, le système pronominal chinois a gardé certaines tendances, issues du chinois classique, dont celle à privilégier les ellipses. Par conséquent, par rapport aux langues européennes telles que le français, le mandarin se distingue par sa prédilection pour les anaphores zéro (voir Li et Thompson, 1979; Chan, 1985).

Pour finir, comparés à leurs équivalents en français, les pronoms personnels de la troisième personne en mandarin ont ceci de particulier: ils apparaissent rarement en position sujet pour désigner des objets inanimés, notamment à l'oral (voir Chao, 1968; Zhu, 1982). Selon cette contrainte, dans les textes chinois, les objets inanimés en position sujet seront généralement repris soit par des syntagmes nominaux soit par des formes zéro. On peut donc s'attendre à ce que les pronoms personnels en position sujet soient beaucoup moins nombreux en mandarin qu'en français.

L'analyse ci-dessus a mis en évidence une divergence formelle importante entre les systèmes pronominaux français et chinois, à cause de laquelle la traduction des formes pronominales entre ces deux langues devra être très sensible aux variations, que nous abordons brièvement ci-après, en donnant des exemples issus du corpus.

\subsection{Variations de traduction}

La traduction des formes pronominales, toute simple qu'elle paraisse, pose des problèmes épineux (Loaiciga Sanchez, 2017) surtout quand il s'agit de langues typologiquement très éloignées. Cela s'explique d'une part par le fait que la catégorie pronominale est dans l'ensemble parmi les plus sensibles aux variations de traduction (Loaiciga Sanchez, 2017), et d'autre part par la nécessité de prendre en compte les différences morphosyntaxiques entre la LS (langue source) et la LC (langue cible).

L'examen soigneux des textes traduits entre le français et le mandarin dans le corpus nous a permis de constater l'apport constant des modifications formelles dans les traductions. D’une part, une référence pronominale employée dans la LS 
n'est pas toujours traduite par son équivalent dans la LC. Plus précisément, un pronom est susceptible d'être traduit par un pronom appartenant à une catégorie différente comme en [1] (le pronom personnel 他们 tāmen «ils» en mandarin est traduit par le pronom démonstratif «ce» en français), par un syntagme nominal comme en [2] (le pronom neutre «le» en français est traduit par le syntagme nominal 这一点 zhè yìdiăn «ce point» en mandarin) ou d'être entièrement omis comme en [3].

[1] LS: [...] 他们是一伙恐怖分子 [...]

[...] tāmen shì yīhuǒ köngbù fènzǐ [...]

[...] 3PL être un-CL terroriste [...]

LC: $[\ldots]$ c'est un groupe de terroristes $[\ldots]$

[2] LS: Nous le devons à nos enfants et aux générations futures.

LC: 为我们的子孙后代应该做到这一点。

Weì wòmen de zišunnbòudài yìnggāi zuò-dào zhè yìdiăn.

pour 2PL-DE² descendant devoir faire-atteindre DEM un point

[3] LS: Le dialogue stratégique franco-chinois est [...] Il constitue la principale plateforme d'échanges politiques du partenariat franco-chinois.

LC：法中战略对话是 [...] Ø是法中合作伙伴之间进行政治交流的主要平 台。

Făzhōng zbànluè duìbuà shì [...] Øshì fãzhōng hézuò huöbàn zbïjiän jìnxíng zbèngzbì jiāoliú de zhǔyào pingtái.

France Chine stratégie dialogue être [...] Øêtre France Chine partenariat entre effectuer politique communication DE principal plate-forme

D'autre part, un syntagme non pronominal dans la LS peut être traduit par un pronom dans la LC comme en [4] (le syntagme nominal 都市女性 düshi nǚxing «femme urbaine» en mandarin est traduit par le pronom personnel «elles» en français), et un pronom qui est absent dans la LS peut être ajouté dans la LC comme [5].

[4] LS： 40,7\%的无子女都市女性的收入高于她们的丈夫 [...]

$40,7 \%$ de wú zǐnŭ dūshì nüxing de shoürù gāoyú tāmen de zbàngfü [...]

$40,7 \%$ DE NEG enfant femme urbaine DE revenu supérieur 3PL-DE mari

LC: $40,7 \%$ d'entre elles qui n'ont pas encore d'enfants gagnent plus que leur mari $[\ldots]$

2. La particule 的 de en mandarin indique la détermination (déterminant $+d e+$ déterminé) ou la possession (possesseur $+d e+$ possédé). 


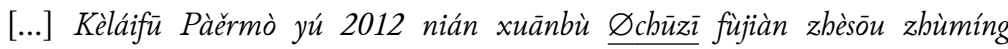
yóulún $[\ldots]$

[...] Clive Palmer en 2012 année déclarer Øreconstruire DEM-CL célèbre paquebot $[\ldots]$

LC: [...] Clive Palmer, a déclaré en 2012 qu'il allait reconstruire ce paquebot célèbre $[\ldots]$

Les exemples ci-dessus montrent déjà un certain degré d'asymétrie entre le français et le mandarin en ce qui concerne l'emploi des références pronominales: celles-ci sont plus fréquentes dans les textes en français qu'en mandarin. Comme nous pouvons le constater, cette asymétrie est due au fait que la traduction du mandarin vers le français a une nette tendance à la pronominalisation (c'est-à-dire au remplacement d'un syntagme par un pronom) comme en [4] et à l'explicitation d'une relation référentielle inexprimée dans la LS comme en [5], alors que celle du français vers le mandarin a plutôt tendance à remplacer un pronom par un syntagme nominal comme en [2] et à rendre implicite l'information référentielle exprimée dans la LS comme en [3]. Somme toute, il s'avère que la traduction pronominale entre le français et le mandarin se caractérise par de nombreuses modifications formelles dans les textes traduits.

Pour mieux observer et analyser les tendances différentes dans les deux directions de traduction, nous avons codé les références pronominales relevées dans le corpus selon les quatre procédés suivants (voir Vinay et Darbelnet, 1958; Guidère, 2016) : i) l'équivalence (qui consiste à traduire une référence pronominale dans la LS par son équivalent dans la LC); ii) la substitution (qui consiste soit à traduire une référence pronominale dans la LS par une référence pronominale de type différent dans la LC comme en [1] ou par une référence nominale comme en [2], soit à remplacer une référence nominale dans la LS par une référence pronominale dans la LC); iii) l'adjonction (qui consiste à rendre explicite une référence pronominale dans la LC); iv) l'omission (qui consiste à rendre implicite une référence pronominale dans la LC).

\section{3. Étude empirique basée sur un corpus de traductions}

En suivant la méthodologie d'analyse contrastive des données de Sun et Grisot (2019), nous avons effectué d'abord des analyses monolingues visant à présenter des faits observés dans chacune des deux langues et à vérifier s'il existe des différences significatives entre textes traduits et textes originaux, avant de proposer une analyse interlinguistique pour examiner et comparer les procédés de traduction adoptés dans les deux directions.

\subsection{Description du corpus et codage des données}

Le corpus parallèle de traductions bidirectionnelles que nous avons dépouillé pour mener cette étude est composé de deux sous-corpus, dont le premier est 
constitué de textes authentiques traduits du français vers le mandarin (françaismandarin) et le deuxième de textes authentiques traduits du mandarin vers le français (mandarin-français). Tous les textes ont été choisis de manière aléatoire sur des sites gouvernementaux ou journalistiques de France et de Chine. Pour le corpus mandarin-français, les textes ont été collectés depuis les sites de presse étatique tels que 人民网 Rénmín wăng «Quotidien du Peuple en ligne» et 新华 网 Xìnbuá wăng «Agence de presse Xinhua». Pour le corpus français-mandarin, les textes ont été collectés essentiellement sur le site de l'ambassade de France en Chine.

Dans le corpus, il y a 45 textes bilingues de longueur différente, dont 30 textes sont traduits du mandarin vers le français et 15 sont traduits du français vers le mandarin. Le corpus mandarin-français comprend environ 14250 mots en mandarin LS et 9950 en français LC, alors que celui du français-mandarin comprend environ 10700 mots en mandarin LC et 6750 en français LS. Quant aux unités référentielles à étudier, nous avons identifié 317 paires dans le corpus mandarin-français et 291 dans le corpus français-mandarin. L'alignement a été fait au niveau phrastique et de manière automatique via le site de traitement multilingue Tmxmall, alors que les données ont été codées de façon manuelle pour pouvoir identifier, dans les deux directions de traduction, les informations suivantes:

1. les références pronominales en français LS et LC ainsi que leur correspondance en mandarin LC et LS;

2. les références pronominales en mandarin LS et LC ainsi que leur correspondance en français LC et LS;

3. le type des références identifiées (référence pronominale, nominale, zéro ou autre ${ }^{3}$; type de référence pronominale comme pronom ou déterminant possessif ${ }^{4}$ ) dans les deux langues;

4. les procédés de traduction (équivalence, substitution, omission, adjonction);

5. la nature du référent: humain ou non humain;

6. le type des pronoms personnels identifiés en français LS et LC (sujet, COD, COI, tonique, adverbial, neutre).

Les données ainsi codées nous ont permis d'effectuer des analyses quantitatives assez précises tant du point de vue monolingue qu'interlinguistique. L'objectif de ces analyses est de tester les hypothèses de recherche préalablement élaborées pour cette étude, que nous présentons ci-après.

3. Quand une référence pronominale identifiée dans la LS est traduite par une expression non référentielle dans la LC.

4. Nous suivons Adam (2005: 89) pour inclure les syntagmes nominaux possessifs dans la catégorie des références pronominales. Selon lui, les déterminants possessifs permettent de rattacher anaphoriquement les syntagmes nominaux à leur antécédent. 


\subsection{Hypothèses de recherche}

Les hypothèses de recherche ont été élaborées selon l'état de nos connaissances sur les deux langues et la thèse que l'interférence se produit inéluctablement lorsque deux langues sont en contact. Si, idéalement, «la traduction consiste à produire dans la langue cible l'équivalent le plus proche et le plus naturel du message de la langue source» (Nida et Taber, 1969: 12), tout traducteur subit, dans une plus ou moins grande mesure, l'influence de la langue source dans ses choix linguistiques (Loock, 2016). Comme l’a montré Jiménez-Crespo (2010) dans une étude basée sur corpus, la fréquence des pronoms est plus élevée en espagnol LC (traduit de l'anglais) qu'en espagnol LS. Selon l'étude empirique de Rabadán et al. (2009), l'antéposition de l'adjectif est plus fréquente dans les textes en espagnol LC (traduit de l'anglais) qu'en espagnol LS (voir aussi Loock, 2016: 193). Nous supposons alors que les textes en LS et en LC s'avèrent significativement différents en termes de fréquence des expressions pronominales.

Dans le même temps, pour reproduire le message de la manière la plus naturelle possible, un bon traducteur s'efforce, que ce soit consciemment ou inconsciemment, de résister à l'interférence de la LS en recourant à divers procédés de variation tels que la substitution, l'omission ou l'adjonction. Compte tenu des caractéristiques propres à chaque langue, nous prédisons que les deux directions de traduction s'avèrent significativement différentes à l'égard des procédés de traduction adoptés.

Partant de toutes ces réflexions, nous avons formulé les hypothèses ci-dessous, dont les quatre premières pour les analyses monolingues et les quatre dernières pour l'analyse interlinguistique:

1. le français LS et LC est caractérisé par une forte fréquence des références pronominales;

2. le mandarin LS et LC est caractérisé par une forte fréquence des ellipses;

3. les références pronominales sont moins fréquentes en français LC qu'en français LS en raison de l'influence de la structure du mandarin LS;

4. les références pronominales sont plus fréquentes en mandarin LC qu'en mandarin LS en raison de l'influence de la structure du français LS;

5. les références pronominales dénombrées dans la partie en français du corpus sont beaucoup plus nombreuses que celles dans la partie en mandarin;

6. le nombre des variations (substitution, adjonction et omission) représente un pourcentage assez important dans les traductions, que ce soit du français vers le mandarin ou du mandarin vers le français;

7. les références pronominales omises sont plus fréquentes dans les traductions $\mathrm{du}$ français vers le mandarin que dans celles du mandarin vers le français;

8. les références pronominales ajoutées sont moins fréquentes dans les traductions du français vers le mandarin que dans celles du mandarin vers le français. 
Les résultats des analyses quantitatives menées pour tester les hypothèses ci-dessus sont présentés dans les deux sous-sections suivantes. Nous fournissons en premier lieu les résultats d'analyses monolingues avant de passer à l'analyse interlinguistique.

\subsection{Analyses monolingues}

Les analyses monolingues ont été faites premièrement dans la partie en français LS et LC du corpus, et deuxièmement, dans la partie en mandarin LS et LC. L'objectif est de donner un premier aperçu quantitatif sur la place des références pronominales dans les deux langues par rapport à d'autres types référentiels (nominal, zéro) et de vérifier, par le biais de comparaisons intralangagières, si la langue source exerce une influence significative sur les textes traduits.

\subsubsection{Résultats en français}

L'analyse monolingue de la partie en français du corpus a montré que les références pronominales sont très fréquentes tant en français LS que LC. Comme l'indiquent les résultats récapitulés dans le tableau 2, parmi les unités relevées, se trouvent $273(93,8 \%)$ références pronominales en français LS et 290 (91,5\%) en français LC. Un test du khi-carré effectué sur les données a confirmé que la différence entre le français LS et LC n'est pas statistiquement significative $\left(\mathrm{X}^{2}=1,204, d f=1\right.$, $p$-value $=0,2726$ ).

\begin{tabular}{|l|l|l|}
\hline Types référentiels & Français LS & Français LC \\
\hline Référence pronominale & $273(93,8 \%)$ & $290(91,5 \%)$ \\
\hline Référence nominale & $3(1 \%)$ & $14(4,4 \%)$ \\
\hline Ellipse & $15(5,2 \%)$ & $12(3,8 \%)$ \\
\hline Autre & $0(0 \%)$ & $1(0,3 \%)$ \\
\hline Total & $291(100 \%)$ & $317(100 \%)$ \\
\hline
\end{tabular}

Tableau 2 - Distribution des types référentiels en français LS et LC

Les références pronominales identifiées en français LS sont divisées, comme l'illustre la figure 1 , en parts quasi égales entre pronoms et déterminants possessifs: $53,5 \%(146 / 273)$ des références identifiées sont réalisées par un pronom alors que 46,5\% (127/273) le sont par un déterminant possessif. L'écart entre ces deux types référentiels en français LS n'est pas statistiquement significatif $\left(\mathrm{X}^{2}=2,645\right.$, $d f=1, p$-value $=0,1039)$. Quant au français LC, 59,7 \% (173/290) des références sont réalisées par un pronom alors que 40,3\% (117/290) le sont par un déterminant possessif, avec une différence statistiquement significative $\left(\mathrm{X}^{2}=21,63, d f=1\right.$, $p$-value $<0,0001)$. 


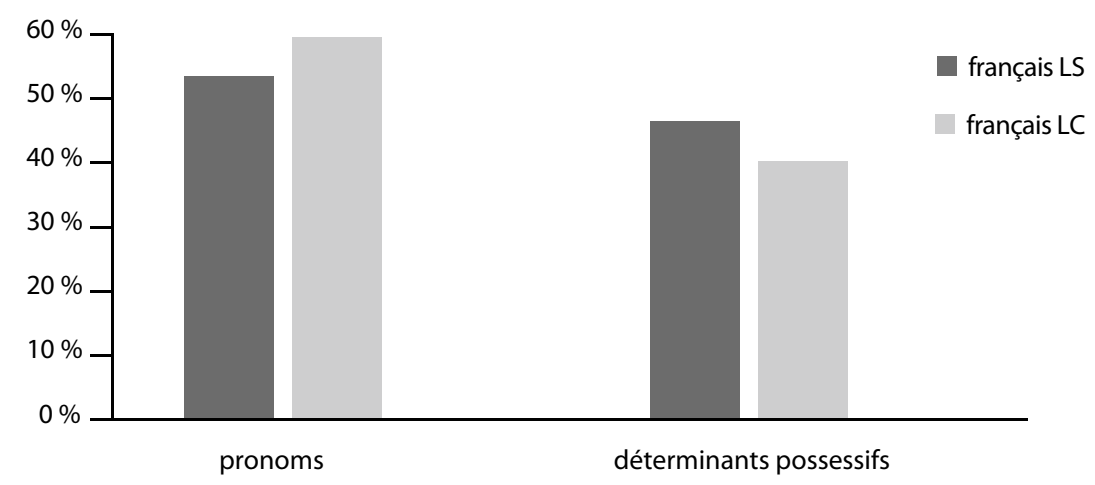

Figure 1 - Distribution des types de référence pronominale en français LS et LC

Les références nominales (traduites depuis ou par des références pronominales) sont peu nombreuses tant en français LS que LC: 3 (1\%) occurrences en français LS par rapport à $14(4,4 \%)$ en français $\mathrm{LC}$, suggérant que les références nominales sont significativement plus fréquentes en français LC qu'en français LS $\left(\mathrm{X}^{2}=6,398, d f=1\right.$, $p$-value $=0,0114)$. En ce qui concerne les ellipses, comme l'indique le tableau 2 , elles représentent 5,2 \% (15/291) en français LS et 3,8 \% (12/317) en français LC, sans différence statistiquement significative $\left(\mathrm{X}^{2}=0,6702, d f=1, p\right.$-value $\left.=0,413\right)$. Pour finir, un test du khi-carré, effectué selon les résultats du tableau 2 , a confirmé que la distribution des types référentiels en français LC est significativement différente de celle en français $\mathrm{LS}\left(\mathrm{X}^{2}=7,867, d f=3, p\right.$-value $\left.=0,0488\right)$.

\subsubsection{Résultats en mandarin}

L'analyse monolingue de la partie en mandarin a été effectuée de la même manière que nous l'avons fait pour celle en français. Dans l'ensemble, par rapport au français, le mandarin recourt beaucoup moins fréquemment aux références pronominales, mais bien plus fréquemment aux références nominales et aux ellipses.

Intéressons-nous en premier lieu aux références pronominales. Comme l'illustre le tableau 3, tant en mandarin LS que LC, leur fréquence n'est pas dominante: $119(37,5 \%)$ en mandarin LS et $107(36,8 \%)$ en mandarin LC, sans différence statistiquement significative $\left(X^{2}=0,0385, d f=1, p\right.$-value $\left.=0,8445\right)$.

Parmi les références pronominales relevées, comme l'illustre la figure 2 , celles réalisées par un pronom représentent 63,9\% (76/119) en mandarin LS et $72 \%(77 / 107)$ en mandarin LC, alors que celles réalisées par un déterminant possessif représentent $36,1 \%(43 / 119)$ en mandarin LS et $28 \%(30 / 107)$ en mandarin LC, indiquant une augmentation de la fréquence des pronoms et une baisse de celle des possessifs en mandarin LC par rapport au mandarin LS (mais sans différence significative: $\mathrm{X}^{2}=1,689$, $d f=1, p$-value $=0,1937)$. Tant pour le mandarin LS que LC, l'écart entre ces deux types référentiels en termes d'occurrences est statistiquement significatif $\left(\mathrm{X}^{2}=18,3\right.$, $d f=1, p$-value $<0,0001$ pour LS; $X^{2}=41,29, d f=1, p$-value $<0,0001$ pour LC). 


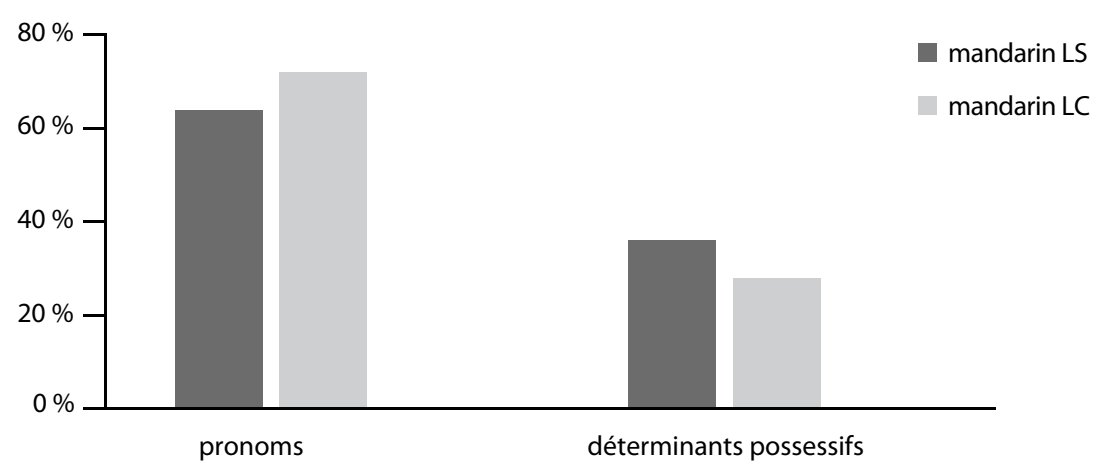

Figure 2 - Distribution des types de référence pronominale en mandarin LS et LC

\begin{tabular}{|l|l|l|}
\hline Types référentiels & Mandarin LS & Mandarin LC \\
\hline Référence pronominale & $119(37,5 \%)$ & $107(36,8 \%)$ \\
\hline Référence nominale & $49(15,5 \%)$ & $83(28,5 \%)$ \\
\hline Ellipse & $142(44,8 \%)$ & $87(29,9 \%)$ \\
\hline Autre & $7(2,2 \%)$ & $14(4,8 \%)$ \\
\hline Total & $317(100 \%)$ & $291(100 \%)$ \\
\hline
\end{tabular}

Tableau 3 - Distribution des types référentiels en mandarin LS et LC

Concernant les références nominales, qui sont traduites soit depuis des références pronominales comme en [2] soit par des références pronominales comme en [4], elles représentent un pourcentage de 15,5\% (49/317) en mandarin LS et 28,5 \% (83/291) en mandarin LC, suggérant que les références nominales sont significativement plus fréquentes en mandarin LC qu'en mandarin LS ( $\mathrm{X}^{2}=15,24, d f=1$, $p$-value $\left.<0,0001\right)$.

Quant aux ellipses, elles représentent 44,8 \% (142/317) en mandarin LS et $29,9 \%(87 / 291)$ en mandarin LC. La différence entre LS et LC est statistiquement significative $\left(\mathrm{X}^{2}=14,34, d f=1, p\right.$-value $\left.=0,0002\right)$, indiquant que les références pronominales sont plus fréquemment omises en mandarin LS qu'en mandarin LC.

Pour finir, un test du khi-carré, effectué selon les résultats du tableau 3, a confirmé que la différence entre le mandarin LC et LS à l'égard de la distribution des types référentiels est statistiquement significative $\left(\mathrm{X}^{2}=23,87, d f=3, p\right.$-value $\left.<0,0001\right)$.

\subsubsection{Discussion}

La première hypothèse pour l'analyse monolingue prédisait que le français LS et LC est caractérisé par une forte fréquence des références pronominales. L'analyse 
quantitative de la partie en français du corpus a bien confirmé cette hypothèse, en montrant que la fréquence de ce type référentiel est prédominante $(93,8 \%$ en LS et $91,5 \%$ en LC) par rapport aux références nominales ( $1 \%$ en LS et $4,4 \%$ en LC) et aux ellipses (5,2\% en LS et 3,8 \% en LC).

La deuxième hypothèse prédisait une forte fréquence des ellipses en mandarin LS et LC. Conformément à nos attentes, le mandarin s'avère avoir une nette préférence pour les ellipses (44,8 \% en LS et $29,9 \%$ en LC) par rapport au français (5,2\% en LS et 3,8\% en LC). De tels résultats empiriques sont en accord avec des études antérieures qui ont souligné la prédilection pour les ellipses (ou anaphores zéro) en mandarin, surtout quand il s'agit de la troisième personne (Tai, 1978; Li et Thompson, 1979; Chan, 1985; Tomlin et Pu, 1991; Christensen, 2000; Hsu, 2019).

Les troisième et quatrième hypothèses visaient à explorer l'interférence de la langue source sur la langue cible. Selon les études en traductologie de corpus (Baker, 1993; Gellerstam, 1986; Loock, 2016), nous aurions attendu une fréquence des références pronominales moins importante en français LC comparé au français LS, ainsi qu'une fréquence des références pronominales plus importante en mandarin LC comparé au mandarin LS. Or, selon nos données, la fréquence des références pronominales en français LC est similaire à celle en français LS (91,5 \% comparés à 93,8 \%; $\mathrm{X}^{2}=1,204, d f=1, p$-value $\left.=0,2726\right)$, suggérant que l'omniprésence des ellipses en mandarin n'a pas exercé une influence significative sur le choix référentiel dans les textes traduits en français. Quant au mandarin, les références pronominales identifiées en mandarin LC ne sont pas significativement plus nombreuses que celles en mandarin LS (36,8 \% comparés à $37,5 \% ; \mathrm{X}^{2}=0,0385, d f=1, p$-value $\left.=0,8445\right)$. Selon ce résultat, la présence importante des références pronominales en français ne semble pas non plus avoir influencé significativement la traduction en mandarin.

Par contre, par le biais de plusieurs comparaisons intralangagières (tant entre français original et traduit qu'entre mandarin original et traduit), nous avons obtenu des résultats au-delà de nos attentes, qui révélaient l'influence de la langue source sur les textes traduits: i) la fréquence des références nominales est significativement plus élevée dans les textes en LC qu'en LS, que ce soit pour le français $\left(\mathrm{X}^{2}=6,398, d f=1, p\right.$-value $\left.=0,0114\right)$ ou le mandarin $\left(\mathrm{X}^{2}=15,24\right.$, $d f=1, p$-value $<0,0001)$; ii) l'écart entre la fréquence des pronoms et celle des déterminants possessifs en français n'est statistiquement significatif que dans les textes en français $\mathrm{LC}\left(\mathrm{X}^{2}=21,63, d f=1, p\right.$-value $<0,0001$ pour le français $\mathrm{LC}$; $\mathrm{X}^{2}=2,645, d f=1, p$-value $=0,1039$ pour le français LS); iii) la fréquence des ellipses est significativement moins importante en mandarin LC qu'en mandarin LS $\left(X^{2}=14,34, d f=1, p\right.$-value $\left.=0,0002\right)$. Ces résultats mettent en lumière des différences significatives entre langue cible et langue source, ce qui est en accord avec les observations de plusieurs études qui ont souligné l'influence de la langue source sur la traduction de certaines expressions linguistiques telles que les pronoms (Jiménez-Crespo, 2010). 
Pour finir, des tests du khi-carré ont également montré que la distribution des types référentiels dans les textes traduits diffère significativement de celle dans les textes originaux, tant pour le français $\left(\mathrm{X}^{2}=7,867, d f=3, p\right.$-value $\left.=0,0488\right)$ que pour le mandarin $\left(\mathrm{X}^{2}=23,87, d f=3, p_{\text {-value }}<0,0001\right)$.

\subsection{Analyse interlinguistique}

Dans le cadre de l'analyse interlinguistique, nous proposons une comparaison quantitative des références pronominales identifiées en français et en mandarin, avant d'analyser les procédés adoptés dans les deux directions de traduction. L'objectif est d'estimer si une disparité significative existe entre ces deux langues à l'égard du choix référentiel, et d'examiner les tendances de traduction dans les deux directions.

\subsubsection{Fréquence des références pronominales}

Selon les données récapitulées dans les tableaux 2 et 3 (voir sections 3.3.1 et 3.3.2), il s'avère que les références pronominales sont significativement plus fréquentes en français qu'en mandarin. Dans les textes traduits du français vers le mandarin, nous avons identifié $273(93,8 \%)$ occurrences du côté français LS par rapport à $107(36,8 \%)$ du côté mandarin LC $\left(X^{2}=208,9, d f=1, p\right.$-value $\left.<0,0001\right)$. Il en est de même pour les textes traduits du mandarin vers le français, dans lesquels $290(91,5 \%)$ occurrences ont été identifiées du côté français LC par rapport à $119(37,5 \%)$ du côté mandarin LS $\left(X^{2}=201,5, d f=1, p\right.$-value $\left.<0,0001\right)$. Au total, comme l'illustre la figure 3, les références pronominales (RPs) identifiées dans la partie en français sont à peu près 2,5 fois plus fréquentes que celles retrouvées en mandarin.

La fréquence moins élevée des références pronominales en mandarin (comparé au français) est compensée, comme l'indique la figure 3, par l'augmentation de la fréquence des références nominales (RNs) et des ellipses. Comme nous l'avons montré plus haut dans les tableaux 2 et 3 , nous avons identifié dans le corpus $49(15,5 \%)$ références nominales du côté mandarin LS par rapport à $14(4,4 \%)$ du côté français LC $\left(\mathrm{X}^{2}=21,59, d f=1, p\right.$-value $\left.<0,0001\right)$, et $142(44,8 \%)$ ellipses du côté mandarin LS par rapport à $12(3,8 \%)$ du côté français LC $\left(X^{2}=144,9\right.$, $d f=1, p$-value $<0,0001)$. Des différences significatives ont été également observées entre les textes en français LS et ceux traduits en mandarin LC.

Les comparaisons interlinguistiques effectuées ci-dessus suggèrent qu'une grande partie des références pronominales dans les textes originaux en français n'aient pas été traduites par leur équivalent en mandarin, et qu'un grand nombre de références nominales ou d'ellipses dans les textes originaux en mandarin aient été traduites par des références pronominales en français. En d'autres termes, les variations de traduction devraient représenter, conformément à ce que prédisait l'hypothèse 6, un pourcentage important dans les traductions. Reste à savoir dans quelle mesure les formes référentielles n'ont pas été «fidèlement» traduites. Pour ce faire, nous avons effectué des analyses plus précises dont les résultats sont présentés ci-après. 


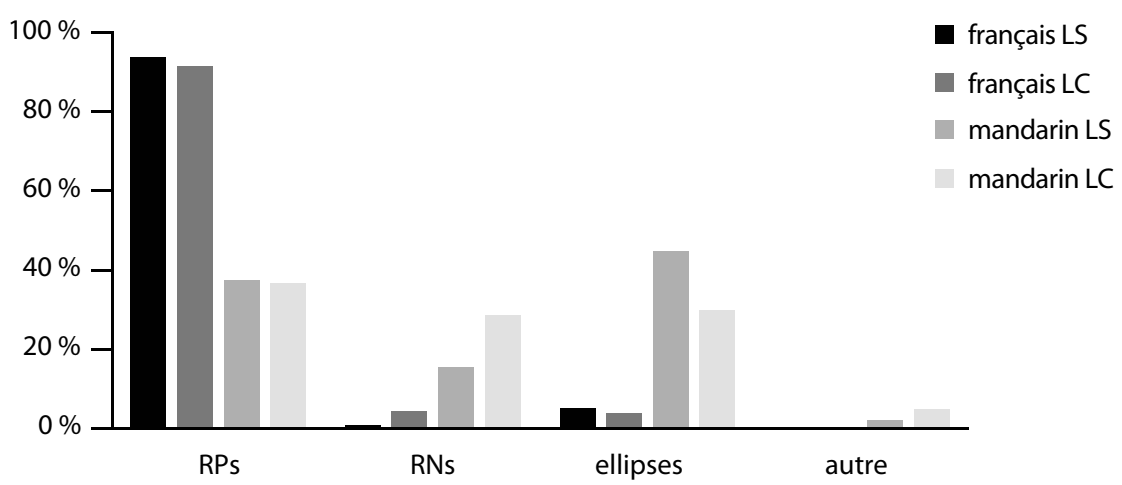

Figure 3 - Distribution des types référentiels en français et en mandarin

\subsubsection{Analyse des procédés de traduction}

La figure 4 illustre la distribution des procédés de traduction (équivalence, substitution, omission et adjonction) dans les deux directions de traduction. Dans les grandes lignes, les résultats montrent que les variations (substitution, omission et adjonction) représentent un pourcentage très important : 78,9\% $\%(250 / 317)$ dans la direction du mandarin vers le français et 75,3\% (219/291) dans celle du français vers le mandarin, sans différence statistiquement significative $\left(\mathrm{X}^{2}=1,119, d f=1, p\right.$-value $\left.=0,2901\right)$, montrant une fois encore que le mandarin et le français divergent significativement dans le choix des moyens référentiels.

En revanche, nous avons constaté des tendances différentes dans les deux directions: les références pronominales sont plus susceptibles d'être substituées ou omises du français vers le mandarin que du mandarin vers le français (39,2\% comparés à 29,7\% pour la substitution; 30,9\% comparés à 3,8\% pour l'omission); en outre, elles sont beaucoup moins fréquemment ajoutées du français vers le mandarin que du mandarin vers le français $(5,2 \%$ comparés à $45,4 \%)$. La distribution des procédés de traduction du mandarin vers le français est significativement différente de celle dans le sens inverse $\left(\mathrm{X}^{2}=165,6, d f=3\right.$, $p$-value $\left.<0,0001\right)$. Cela explique pour une grande part la disparité importante entre le français et le mandarin en termes d'occurrences de références pronominales.

Examinons maintenant de plus près les procédés de traduction. Selon les données du tableau 4, les pronoms ayant une fonction sujet en mandarin $(50,7 \%)$ sont traduits plus fréquemment par leur équivalent en français par rapport aux pronoms occupant une fonction non sujet $(19,4 \%)$ et aux déterminants possessifs $(29,9 \%)$.

Dans la direction du français vers le mandarin, nous retrouvons la prédominance des pronoms en position sujet: ceux-ci représentent, comme l'illustre le tableau 5, 69,4\% des traductions équivalentes. Pourtant, la différence entre les deux directions de traduction est statistiquement significative $\left(\mathrm{X}^{2}=5,074, d f=1\right.$, $p$-value $=0,0243)$. 


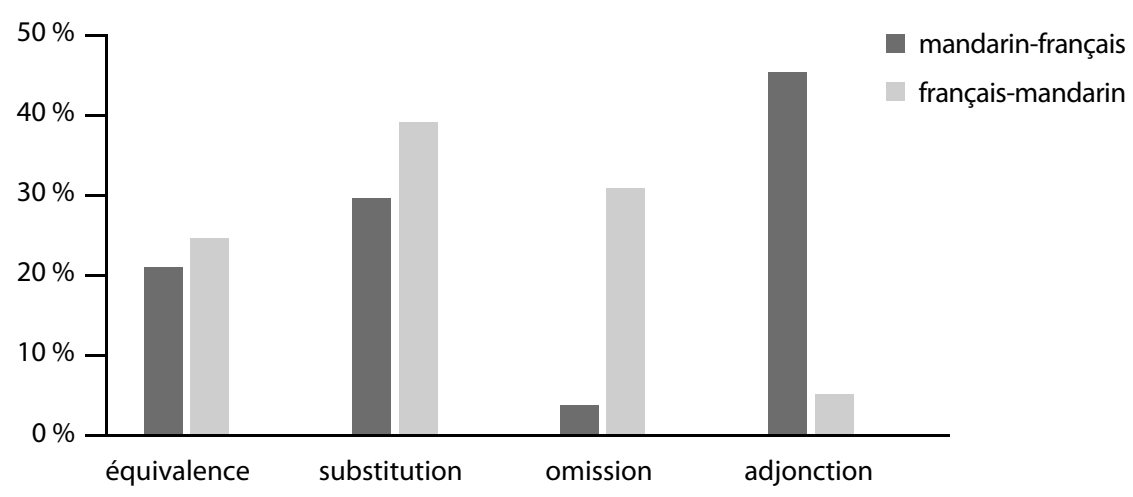

Figure 4-Distribution des procédés de traduction dans les deux directions

\begin{tabular}{|l|l|l|l|l|}
\hline Types & Équivalence & Substitution & Adjonction & Omission \\
\hline Pronom en position sujet & $34(50,7 \%)$ & $14(14,9 \%)$ & $55(38,2 \%)$ & $4(33,3 \%)$ \\
\hline Pronom en position non sujet & $13(19,4 \%)$ & $9(9,6 \%)$ & $17(11,8 \%)$ & $2(16,7 \%)$ \\
\hline Déterminant possessif & $20(29,9 \%)$ & $17(18,1 \%)$ & $72(50 \%)$ & $6(50 \%)$ \\
\hline Syntagme nominal & - & $49(52,1 \%)$ & - & - \\
\hline $\begin{array}{l}\text { Autre (syntagme verbal, } \\
\text { adverbe, etc.) }\end{array}$ & - & $5(5,3 \%)$ & - & - \\
\hline Total & $67(100 \%)$ & $94(100 \%)$ & $144(100 \%)$ & $12(100 \%)$ \\
\hline
\end{tabular}

Tableau 4 - Procédés de traduction du mandarin vers le français

\begin{tabular}{|l|l|l|l|l|}
\hline Types & Équivalence & Substitution & Adjonction & Omission \\
\hline Pronom en position sujet & $50(69,4 \%)$ & $35(30,7 \%)$ & $9(60 \%)$ & $32(35,6 \%)$ \\
\hline Pronom en position non sujet & $1(1,4 \%)$ & $18(15,8 \%)$ & $2(13,3 \%)$ & $10(11,1 \%)$ \\
\hline Déterminant possessif & $21(29,2 \%)$ & $58(50,9 \%)$ & $4(26,7 \%)$ & $48(53,3 \%)$ \\
\hline Syntagme nominal & - & $3(2,6 \%)$ & - & - \\
\hline Total & $72(100 \%)$ & $114(100 \%)$ & $15(100 \%)$ & $90(100 \%)$ \\
\hline
\end{tabular}

Tableau 5 - Procédés de traduction du français vers le mandarin

Par ailleurs, en faisant une analyse plus approfondie, nous avons constaté que tant pour la direction du mandarin vers le français que pour celle du français vers le mandarin, les pronoms (en position sujet ou non sujet) traduits par leur équivalent 
renvoient quasi tous à un référent humain: 95,7 \% (45/47) pour la direction du mandarin vers le français et $98 \%(50 / 51)$ pour celle du français vers le mandarin. Quant aux déterminants possessifs traduits par leur équivalent, nous avons retrouvé des résultats similaires, $90 \%(18 / 20)$ d'entre eux traduits du mandarin vers le français renvoient à des référents humains et 95,2\% (20/21) dans les textes traduits du français vers le mandarin. La différence entre les deux directions n'est pas significative $\left(\mathrm{X}^{2}=0,8563, d f=3, p\right.$-value $\left.=0,8359\right)$. Ces résultats montrent que dans les deux directions, les références pronominales désignant des êtres humains sont beaucoup plus susceptibles d'être «fidèlement» traduites par rapport à celles renvoyant à des entités non humaines.

En ce qui concerne la substitution, d'importantes différences entre les deux directions de traduction peuvent être observées dans les résultats obtenus. Comme nous pouvons le constater dans les tableaux 4 et 5 , du mandarin vers le français, les syntagmes nominaux $(52,1 \%)$ sont plus susceptibles d'être substitués comparés à d'autres types référentiels tels que les pronoms $(24,5 \%)$ et les déterminants possessifs $(18,1 \%)$, alors que du français vers le mandarin, les déterminants possessifs sont les plus fréquents $(50,9 \%)$, suivis des pronoms $(46,5 \%)$ et des syntagmes nominaux $(2,6 \%)$.

En examinant de plus près les syntagmes nominaux substitués par des références pronominales (substitution pronominale) lors du passage du mandarin vers le français, nous avons constaté que ceux qui renvoient à des référents non humains sont plus fréquents que ceux désignant des êtres humains: 67,3\% (33/49) comparés à 32,7 \% (16/49). Quant à la direction du français vers le mandarin, les résultats montrent que les références pronominales sont plus sensibles à la substitution nominale lorsqu'elles réfèrent à des entités non humaines: 81,9\% (68/83) comparés à $18,1 \%(15 / 83)$. Ces résultats montrent que les références nominales (ou pronominales) désignant des entités non humaines sont fortement sensibles à la substitution pronominale (ou nominale) lors du passage du mandarin au français (ou du français au mandarin).

En ce qui concerne le procédé d'adjonction dans la direction du mandarin vers le français, comme l'illustre le tableau 4, parmi les 144 termes référentiels ajoutés dans les textes traduits, nous avons identifié $72(50 \%)$ déterminants possessifs, $55(38,2 \%)$ pronoms en position sujet et $17(11,8 \%)$ pronoms en position non sujet. Quant aux termes omis du français vers le mandarin, nous avons identifié, comme l'illustre le tableau 5, 48 (53,3\%) déterminants possessifs, 32 (35,6\%) pronoms en position sujet et $10(11,1 \%)$ pronoms en position non sujet. L'adjonction (ou l'omission) des déterminants possessifs du mandarin vers le français (ou du français vers le mandarin) montre que le français et le mandarin divergent considérablement à l'égard de l'anaphore possessive: comme l'illustrent les exemples [6] et [7], à la différence du mandarin qui privilégie le déterminant zéro, le français tend à utiliser un déterminant possessif pour signaler la relation coréférentielle entre un syntagme nominal et son antécédent (ou conséquent). 
LS：[...] 都市女性对于 $\varnothing$ 家庭的经济贡献会随子女的出生而降低。

[...] düshì nüxing duìú Øjiätíng de jingjì gòngxiàn buì suí zǐnü de cbūshēng ér jiàngdì

[...] femme urbain pour famille DE économie contribution FUT enfant DE naissance diminuer

LC: [...] cette contribution économique des femmes urbaines pour leur famille peut chuter avec la naissance d'un premier enfant.

[7] LS: À l'occasion de son premier déplacement international en Chine, Mme Fleur Pellerin $[\ldots]$

\section{LC: [...] 福乐尔・佩勒林女士在 $\varnothing$ 访华期间 [...]}

[...] Fúlèèr Pèilelin nǘshì zài Øfănghuá qỉjiān [...]

[...] Fleur Pellerin madame ZAI ${ }^{5}$ visiter Chine pendant [...]

S'agissant des pronoms ajoutés du mandarin vers le français ou omis du français vers le mandarin, nous avons constaté que par rapport à la première ou deuxième personne, la troisième personne représente une proportion beaucoup plus importante. Plus précisément, parmi les pronoms ajoutés du mandarin vers le français, ceux de la troisième personne représentent $73,6 \%(53 / 72)$, dont $67,9 \%(36 / 53)$ renvoient à des référents humains et $32,1 \%(17 / 53)$ à des référents non humains. Ces résultats montrent que la traduction du mandarin vers le français a tendance à remplacer une anaphore «invisible» (ou zéro) par une reprise pronominale notamment quand celle-là renvoie à un antécédent désignant un être humain. Pourtant, aux pronoms de la troisième personne omis du français vers le mandarin, nous avons constaté le phénomène inverse : 45,8 \% (11/24) désignent des êtres humains alors que $54,2 \%(13 / 24)$ renvoient à des référents non humains. Étant donné la taille limitée de l'échantillon, il serait nécessaire d'élargir le corpus en vue d'une étude plus approfondie.

Nous terminons l'analyse interlinguistique par un examen des pronoms identifiés dans la partie en français du corpus. Selon les résultats obtenus, comme l'illustre le tableau 6, il s'avère que la traduction d'un pronom (implicite ou explicite) est liée à sa position syntaxique: parmi les pronoms qui existent dans les deux langues, ceux en position sujet sont plus susceptibles d'être traduits par leur équivalent (43,1\% pour la direction du français vers le mandarin et $34,6 \%$ pour celle du mandarin vers le français) par rapport à ceux en position non sujet (5,6\% du français vers le mandarin et $24,4 \%$ du mandarin vers le français). Ces derniers sont plutôt sensibles aux variations (substitution: $50 \%$ du français vers le mandarin et $44,4 \%$ du mandarin vers le français; omission: $44,4 \%$ du français vers le mandarin; adjonction: $31,1 \%$ du mandarin vers le français).

5. Zài est considéré en général comme une marque de l'aspect progressif d'une situation (zài + syntagme verbal) ou une préposition pour indiquer le lieu (zài + lieu). Dans cet exemple, la locution zài... qījiāan signifie «pendant la durée de...» en français. 


\begin{tabular}{|c|c|c|c|}
\hline Pronoms en français & $\begin{array}{l}\text { Procédés de } \\
\text { traduction }\end{array}$ & Mandarin LC & Mandarin LS \\
\hline \multirow{5}{*}{ Pronom sujet } & Équivalence & $50(43,1 \%)$ & $36(34,6 \%)$ \\
\hline & Substitution & $34(29,3 \%)$ & $23(22,1 \%)$ \\
\hline & Omission & $32(27,6 \%)$ & - \\
\hline & Adjonction & - & $45(43,3 \%)$ \\
\hline & Total & $116(100 \%)$ & $104(100 \%)$ \\
\hline \multirow{5}{*}{$\begin{array}{l}\text { Pronom } \\
\text { COD/COI/tonique }\end{array}$} & Équivalence & $1(5,6 \%)$ & $11(24,4 \%)$ \\
\hline & Substitution & $9(50 \%)$ & $20(44,4 \%)$ \\
\hline & Omission & $8(44,4 \%)$ & - \\
\hline & Adjonction & - & $14(31,1 \%)$ \\
\hline & Total & $18(100 \%)$ & $45(100 \%)$ \\
\hline \multirow{5}{*}{$\begin{array}{l}\text { Pronom sans équivalent } \\
\text { chinois }\end{array}$} & Équivalence & - & - \\
\hline & Substitution & $10(83,3 \%)$ & $11(45,8 \%)$ \\
\hline & Omission & $2(16,7 \%)$ & - \\
\hline & Adjonction & - & $13(54,2 \%)$ \\
\hline & Total & $12(100 \%)$ & $24(100 \%)$ \\
\hline
\end{tabular}

Tableau 6 - Procédés de traduction par rapport aux pronoms en français LS et LC

En outre, comme l'indique le tableau 6, nous avons également identifié dans les traductions adaptées un certain nombre de pronoms sans équivalents chinois (par exemple, les pronoms adverbiaux «en» et «y», les pronoms neutres «le» et «on»). Cela montre que les variations de traduction ne sont pas indépendantes des caractéristiques intrinsèques de la langue et explique dans une certaine mesure la fréquence moins élevée des formes pronominales en mandarin.

\subsubsection{Discussion}

Comme le prédisait la cinquième hypothèse, notre analyse interlinguistique a montré, en premier lieu, que les références pronominales dans la partie en français du corpus sont significativement plus nombreuses que celles dans la partie en mandarin : 93,8 \% en français LS contre 36,8 \% en mandarin LC $\left(\mathrm{X}^{2}=208,9, d f=1, p\right.$-value $\left.<0,0001\right)$, et $91,5 \%$ en français LC contre $37,5 \%$ en mandarin LS $\left(\mathrm{X}^{2}=201,5, d f=1\right.$, $p$-value $<0,0001)$. La baisse de la fréquence des références pronominales dans la partie en mandarin a été contrebalancée par une nette augmentation des références nominales ( $15,5 \%$ en mandarin LS contre $4,4 \%$ en français LC) et des ellipses (44,8\% en mandarin LS contre 3,8 \% en français LC). 
Conformément à la remarque de Loaiciga Sanchez (2017), notre analyse a montré que la catégorie des pronoms est fortement sensible aux variations de traduction: l'analyse des procédés de traduction a mis en évidence une fréquence importante des variations tant dans les textes traduits du mandarin vers le français $(78,9 \%)$ que dans ceux traduits du français vers le mandarin $(75,3 \%)$. Aucune différence significative n'étant observée $\left(\mathrm{X}^{2}=1,119, d f=1, p\right.$-value $\left.=0,2901\right)$, la sixième hypothèse a été validée. Nous avons pourtant observé des différences importantes entre les deux directions de traduction: le procédé d'omission s'avère plus fréquemment adopté du français vers le mandarin $(30,9 \%)$ que du mandarin vers le français $(3,8 \%)$, tandis que c'est complètement l'inverse pour le procédé d'adjonction (5,2\% contre 45,4\%). Ces résultats, qui ont confirmé les deux dernières hypothèses, sont compatibles avec plusieurs études antérieures soulignant l'omniprésence des ellipses anaphoriques en mandarin (Li et Thompson, 1979; Chan, 1985; Christensen, 2000; Hsu, 2019), et expliquent le déséquilibre significatif entre la partie en français du corpus et celle en mandarin à l'égard de la fréquence des références pronominales.

Les résultats ci-dessus exposés ont mis en évidence une nette tendance à privilégier les références pronominales en français, en contraste frappant avec le mandarin qui a une prédilection plutôt pour les reprises nominales et les ellipses. Une telle divergence peut s'expliquer en premier lieu par le fait que le mandarin permet couramment l'occurrence d'un pronom nul en position sujet et à la fois en position objet (Huang, 1984; voir aussi Zribi-Hertz, 1996) et l'omission d'un déterminant possessif (Chao, 1968) comme en [6] et [7], alors que le français ne l'accepte que dans des contextes restreints (Zribi-Hertz, 1996), par exemple, dans la deuxième proposition juxtaposée. Ainsi, du point de vue économique, pour éviter des répétitions redondantes, le mandarin tend à omettre les pronoms ou déterminants pourvu que leur omission ne rende pas la phrase agrammaticale ou ambiguë, ainsi produisant à moindres coûts des effets contextuels plus riches (Sperber et Wilson, 1989).

Pourtant, comment pourrait-on expliquer la fréquence importante des reprises nominales dans la partie en mandarin du corpus si le recours à une forme pronominale ou zéro est plus économique? Selon nous, cela pourrait s'expliquer d'une part par le fait que les pronoms personnels en mandarin sont utilisés essentiellement pour désigner des personnes ( $\mathrm{Li}$ et Thompson, 1981) et d'autre part par le fait d'éviter de rendre la phrase agrammaticale ou ambiguë. Notre analyse interlinguistique n'a examiné que le premier facteur, mais une étude plus qualitative sera effectuée dans le futur pour la deuxième explication. Selon les résultats obtenus, nous avons pu montrer que la manière de traduire une référence pronominale entre le français et le mandarin est étroitement liée au caractère humain ou non humain de l'entité qu'elle désigne : i) parmi les références pronominales «fidèlement» traduites, celles renvoyant à des référents humains représentent un pourcentage prédominant $(94 \%$ pour la direction du mandarin vers le français et $97,2 \%$ pour celle du français vers le mandarin); ii) par rapport aux syntagmes nominaux désignant des entités humaines, ceux qui renvoient à des entités non humaines sont beaucoup plus 
sensibles à la substitution pronominale du mandarin vers le français $(67,3 \%)$ ou à la substitution nominale du français vers le mandarin (81,9\%); iii) parmi les pronoms de la troisième personne ajoutés du mandarin vers le français, ceux qui renvoient à des référents humains sont plus nombreux $(67,9 \%)$ que ceux désignant des entités non humaines $(32,1 \%)$. Ces résultats font ressortir que le mandarin privilégie les références pronominales et les ellipses pour référer à des êtres humains et les syntagmes nominaux pour désigner des entités non humaines (notamment inanimées). De ce fait, la traduction du français vers le mandarin a tendance à traduire les références pronominales renvoyant à des êtres humains par leur équivalent ou tout simplement à les omettre si leur omission ne rend pas la phrase agrammaticale ou ambiguë, et à remplacer celles référant à des entités non humaines (notamment inanimées) par des syntagmes nominaux.

En plus des résultats exposés ci-dessus, notre analyse interlinguistique a également souligné le lien entre la traduction d'une référence pronominale et la position syntaxique qu'elle occupe. Selon Chao (1968) et Zhu (1982), l'emploi des pronoms de la troisième personne pour désigner les objets est fortement restreint en mandarin et dépendant de la position syntaxique. Par exemple, à l'oral, l'emploi d'un pronom de la troisième personne pour désigner un objet inanimé n'est légitime que lorsque celui-ci est en position non sujet ${ }^{6}$. Selon notre analyse quantitative, parmi les pronoms en position sujet, $43,1 \%$ en français LS et 34,6\% en français LC ont réussi à trouver leur équivalent en mandarin LC et LS. En revanche, les pronoms en position non sujet semblent plus sensibles aux variations de traduction: au total, 94,4\% pour la direction du français vers le mandarin et $75,6 \%$ pour celle du mandarin vers le français. En outre, nous avons également souligné l'apport des modifications dans les traductions en raison de l'absence d'équivalents chinois pour certains pronoms français.

\section{Conclusion}

En nous inscrivant dans la tendance actuelle à privilégier le recours aux corpus pour effectuer des études contrastives (McEnery et al., 2003; Noël et Colleman, 2010; Xiao et McEnery, 2010; Biber et Reppen, 2015), nous avons examiné de manière quantitative et contrastive la référence pronominale dans les traductions entre le français, une langue riche en expressions pronominales, et le mandarin, une langue à morphologie pauvre.

Conformément à nos attentes et aux études antérieures ( $\mathrm{Li}$ et Thompson, 1979; Chan, 1985; Jonasson, 2009), les résultats de nos analyses monolingues ont montré que les textes en français sont marqués par une fréquence importante des références pronominales, alors qu'en mandarin, les reprises nominales et notamment les ellipses sont fortement privilégiées. En comparant les formes référentielles

6. Sous l'influence des langues européennes comme l'anglais (Li et Thompson, 1981), l'occurrence d'un pronom de la troisième personne désignant un objet inanimé est observable en position sujet d'une phrase en mandarin, mais cet emploi non prototypique reste relativement peu fréquent. 
identifiées dans les textes originaux avec celles dans les traductions, nous avons pu mettre en évidence l'influence de la langue source sur le choix référentiel lors du processus de traduction.

Selon les résultats de notre analyse interlinguistique, la catégorie des pronoms a été prouvée fortement sensible aux variations de traduction. Ce problème de la traduction pronominale a été également mis en évidence par Loaiciga Sanchez (2017) qui a fourni des preuves empiriques montrant qu'une référence pronominale dans une langue peut être traduite dans une autre par un syntagme nominal, un pronom de type différent ou une forme zéro. Nos résultats sur la traduction pronominale entre le français et le mandarin sont compatibles avec ses remarques, mais nous avons également pu souligner une autre tendance de traduction, à savoir celle à expliciter dans la langue cible les références pronominales inexprimées dans la langue source.

L'analyse interlinguistique a également identifié plusieurs facteurs susceptibles d'expliquer le nombre important de cas de divergence entre le français et le mandarin en ce qui concerne le choix référentiel: i) l'absence d'équivalence systématique entre les deux langues; ii) l'autorisation des pronoms nuls en mandarin; iii) le caractère humain/non humain ou animé/inanimé du référent; iv) la position syntaxique de la référence pronominale. Plus précisément, le système pronominal étant beaucoup moins riche en mandarin, cette langue ne possède pas d'équivalents pour certains pronoms français et, par conséquent, doit les remplacer par des syntagmes nominaux, des pronoms de type différent ou des formes zéro. Le mandarin autorise les pronoms nuls en position sujet et en position objet, alors qu'en général, le français ne l'accepte pas et, par conséquent, doit les remplacer par des formes référentielles explicites. Le français permet l'occurrence des pronoms inanimés en position sujet, alors qu'en général, le mandarin ne l'accepte pas et, par conséquent, doit les remplacer par des syntagmes nominaux ou tout simplement des formes zéro. Pourtant, au-delà de ces contraintes linguistiques au niveau propositionnel, il serait nécessaire d'explorer dans notre future recherche des facteurs discursifs et pragmatiques pour expliquer d'une manière plus poussée la différence observée par rapport à la référence pronominale en français et en mandarin. Il serait également intéressant de mener des études expérimentales auprès de participants natifs et apprenants pour démêler l'influence de différents facteurs dans l'interprétation de la référence pronominale en français et en mandarin.

\section{Références bibliographiques}

AdAm, J.-M. 1999. Linguistique textuelle: des genres de discours aux textes. Paris: Nathan.

Adam, J.-M. 2005. La linguistique textuelle: introduction à l'analyse textuelle des discours. Paris : A. Colin.

ArIEL, M. 1988. Referring and Accessibility. Journal of Linguistics 24 (1): 65-87.

BAKER, M. 1993. Corpus Linguistics and Translation Studies: Implications and Applications. In M. Baker, G. Francis et E. Tognini-Bonelli (éd.), Text and Tecbnology: In Honour of Jobn Sinclair. Philadelphie - Amsterdam: J. Benjamins: 233-250. 
Biber, D. et Reppen, R. (éd.) 2015. The Cambridge Handbook of English Corpus Linguistics. Cambridge : Cambridge University Press.

Chan, N.-P. 1985. The Use of the Third-Person Pronoun in Mandarin Chinese. Cabiers de linguistique - Asie orientale 14 (1): 47-81.

Chaо, Y.-R. 1968. A Grammar of Spoken Chinese. Berkeley: University of California Press.

Charolles, M. 1978. Introduction aux problèmes de la cohérence des textes. Langue française $38: 7-41$.

Charolles, M. 1995. Cohésion, cohérence et pertinence du discours. Travaux de Linguistique 29: 125-151.

Christensen, M. B. 2000. Anaphoric Reference in Spoken and Written Chinese Narrative Discourse. Journal of Chinese Linguistics 28 (2): 303-336.

Clancy, P. 1980. Referential Choice in English and Japanese Narrative Discourse. In W. L. Chafe (éd.), The Pear Stories. Cognitive, Cultural, and Linguistic Aspects of Narrative Production. Norwood: Ablex Publishing: 127-202.

Cornish, F. 2000. L'accessibilité cognitive des référents, le centrage d'attention et la structuration du discours: une vue d'ensemble. Verbum 22 (1) : 7-30.

Cornish, F., Garnham, A., Cowles, H. W., Fossard, M. et André, V. 2005. Indirect Anaphora in English and French: A Cross-linguistic Study of Pronoun Resolution. Journal of Memory and Language 52 (3): 363-376.

Demol, A. 2008. Les anaphoriques celui-ci et il. Étude des facteurs qui déterminent leur choix. Thèse de doctorat. Université de Gand.

De Mulder, W., Vet, C. et Vetters, C. (éd.) 2001. Anaphores pronominales et nominales: études pragma-sémantiques. Amsterdam - New York: Rodopi.

Gellerstam, M. 1986. Translationese in Swedish Novels Translated from English. In L. Wollin et H. Lindquist (éd.), Translation Studies in Scandinavia. Lund: C. W. K. Gleerup: 88-95.

Grevisse, M. et Goosse, A. 2008. Le bon usage: grammaire française. Bruxelles: De BoeckDuculot [14 éd.].

Guidère, M. 2016. Introduction à la traductologie. Penser la traduction: bier, aujourd'bui, demain. Louvain-la-Neuve: De Boeck.

Halliday, M. A. K. et Hasan, R. 1976. Cohesion in English. Londres: Longman.

Hsu, Y.-Y. 2019. Information Structure in Chinese Discourse. In C. SHEI (éd.), The Routledge Handbook of Chinese Discourse Analysis. Abingdon - New York: Routledge: 130-144.

HuAng, C.-T. J. 1984. On the Distribution and Reference of Empty Pronouns. Linguistic Inquiry 15 (4): 531-574.

JimÉnez-Crespo, M. A. 2010. The Future of Universal Tendencies: A Review of Papers Using Localized Websites. In R. XIAO (éd.), Proceedings of the International Symposium on Using Corpora in Contrastive and Translation Studies. 1-34. En ligne à l'adresse suivante: https://www.lancaster.ac.uk/fass/projects/corpus/UCCTS2010Proceedings/ papers/Jimenez.pdf.

Jonasson, K. 2006. Démonstratifs indéfinis en suédois et leurs correspondants en français. Langue française $152: 24-38$. 
Jonasson, K. 2009. Formes lexicales et pronominales dans des chaînes de référence en français et suédois. Syntaxe et sémantique $10: 111-130$.

KLeIBer, G. 1994. Anaphores et pronoms. Louvain-la-Neuve: Duculot.

Li, C. N. et Thompson, S. A. 1979. Third-Person Pronouns and Zero-Anaphora in Chinese Discourse. In J. P. Kimball et T. Givón (éd.), Syntax and Semantics. New York: Academic Press. Vol. 12: Discourse and Syntax: 311-335.

Li, C. N. et Thompson, S. A. 1981. Mandarin Chinese: A Functional Reference Grammar. Berkeley - Londres: University of California Press.

Loaiciga Sanchez, S. 2017. Pronominal Anaphora and Verbal Tenses in Machine Translation. Thèse de doctorat. Université de Genève.

Loock, R. 2016. La traductologie de corpus. Villeneuve-d'Ascq: Presses universitaires du Septentrion.

LundQuist, L. 2005. Noms, verbes et anaphores (in)fidèles. Pourquoi les Danois sont plus fidèles que les Français. Langue française 145 : 73-91.

Lundquist, L. 2007. Comparing Evolving Anaphors in Danish and French. In I. Korzen et L. Lundquist (éd.), Comparing Anaphors. Between Sentences, Texts and Languages. Copenhague: Samfundslitteratur Press: 111-125.

McEnery, A., Xiao, Z. et Mo, L. 2003. Aspect Marking in English and Chinese: Using the Lancaster Corpus of Mandarin Chinese for Contrastive Language Study. Literary and Linguistic Computing 18 (4): 361-378.

NidA, E. A. et TABER, C. R. 1969. The Theory and Practice of Translation. Leyde: Brill.

NoëL, D. et Colleman, T. 2010. Believe-Type Raising-to-Object and Raising-to-Subject Verbs in English and Dutch: A Contrastive Investigation in Diachronic Construction Grammar. International Journal of Corpus Linguistics 15 (2): 157-182.

Rabadán, R., Labrador, B. et Ramón, N. 2009. Corpus-Based Contrastive Analysis and Translation Universals. A Tool for Translation Quality Assessment English-Spanish. Babel 55 (4): 303-328.

Reboul, A. 1989. Résolution de l'anaphore pronominale: sémantique et/ou pragmatique. Cabiers de linguistique française 10: 77-100.

Reboul, A. 1994. L'anaphore pronominale: le problème de l'attribution des référents. In J. Moeschler, A. Reboul, J.-M. Luscher et J. Jayez (éd.), Langage et pertinence: référence temporelle, anaphore, connecteurs et métaphore. Nancy: Presses universitaires de Nancy: 105-173.

Reboul, A. et Moeschler, J. 1998. Pragmatique du discours: de l'interprétation de l'énoncé à l'interprétation du discours. Paris: A. Colin.

SPERbER, D. et Wilson, D. 1989. La pertinence. Communication et cognition. A. GERSCHENFELD et D. SPERber (trad.). Paris: Minuit.

Sun, J. et Grisot, C. 2019. Le temps dans les traductions français-mandarin et mandarinfrançais: convergence ou divergence? Nouveaux cabiers de linguistique française 33: 11-44.

TAI, H.-Y. J. 1978. Anaphoric Restraints in Mandarin Chinese Narrative Discourse. In J. Hinds (éd.), Anaphora in Discourse. Edmonton: Linguistic Research : 279-338.

Tomlin, R. S. et Pu, M. M. 1991. The Management of Reference in Mandarin Discourse. Cognitive Linguistics 2 (1) : 65-93. 
VANDERBAUWHEDE, G. 2014. L'anaphore démonstrative en français et en néerlandais: étude empirique de cas de divergence et leurs répercussions en L2. In M. FossarD et M.-J. BÉGuELIN (éd.), Nouvelles perspectives sur l'anaphore: points de vue linguistique, psycholinguistique et acquisitionnel. Berne: P. Lang: 287-306.

Vinay, J.-P. et Darbelnet, J. 1958. Stylistique comparée de l'anglais et du français. Paris: Didier.

WANG, L. 1954. Zhongguo yufa lilun [Theory of Chinese Grammar]. Pékin: Zhonghua Book Company.

Xiao, R. et McEnery, T. 2010. Corpus-Based Contrastive Studies of English and Chinese. New York - Londres : Routledge.

Zhu, D.-X. 1982. Yufa jiangyi [Notes on Chinese Grammar]. Pékin: The Commercial Press.

ZRIBI-Hertz, A. 1996. L'anaphore et les pronoms: une introduction à la syntaxe générative. Villeneuve-d'Ascq: Presses universitaires du Septentrion. 